\title{
Progress in Modern Pharmacological Research and Clinical Application of Daihuang Zhechong Pill
}

\author{
Yue Zhao', Zhenyuan $\mathrm{Hu}^{2 *}$ \\ 1Shaanxi University of Chinese Medicine, Xianyang 712000, Shaanxi Province, China \\ ${ }^{2}$ The Affiliated Hospital of Shaanxi University of Chinese Medicine, Xianyang 712000, Shaanxi Province, China \\ *Corresponding author: Zhenyuan Hu, huzhenyuan123@Tom.com
}

\begin{abstract}
Daihuang Zhechong pill is an important prescription in ancient books for the treatment of promoting blood circulation and breaking blood stasis. This paper mainly discusses the pharmacological study and clinical application of Daihuang Zhechong pill.
\end{abstract}

Keywords: Daihuang Zhechong pill; Modern pharmacological research; Clinical application

Publication date: November 2021; Online publication: November 30, 2021

\section{Introduction}

Daihuang Zhechong pill is composed of prescriptions from Synopsis of Golden Chamber: Radix Rehmanniae Preparata, fried ground beetle, fried hirudo, fried June beetle grub, fried Resina Toxicodendri, peach kernel, apricot kernel, Radix Scutellariae, Radix Rehmaniae Recens, Radix Paeoniae Alba, and roasted Radix Glycyrrhizae. As a prescription for the treatment of dry blood internal knot caused by five tuberculosis deficiency, its key points of syndrome differentiation are abdominal distension, poor appetite, dark eyes, pale yellow and dark complexion, tongue ecchymosis and astringent pulse string. Combined with various drugs, there is tonic in the attack, so that blood stasis can be removed, blood stasis and heat can be cleared, Yin blood can be supplemented, and pills can be used to slow down the treatment, so that dried blood can be melted, which is called "there is deficiency in slowing down." With the acquisition of the research results of its various pharmacological effects, its clinical application also has a good curative effect.

\section{Modern pharmacological research of Daihuang Zhechong pill}

\subsection{Pharmacological study of digestive system}

Xudong Liu et al. ${ }^{[1]}$ tried to reveal whether Daihuang Zhechong pill directly acts on LPS-TLR4-MYD88 pathway. Therefore, carbon tetrachloride was used to make an animal model of liver fibrosis. The experimental results show that Daihuang Zhechong pill can indeed act on LPS-TLR4-MYD88 pathway and finally achieve the effect of anti-liver fibrosis. The general clinical progress of chronic liver disease is from liver fibrosis to liver cirrhosis to liver cancer. Therefore, effective control of liver fiber can effectively curb the further spread of liver disease and truly prevent liver disease from the source of the disease.

\subsection{Pharmacological study of digestive system}

Xueying Hui et al. ${ }^{[2]}$ designed experiments for verification. The results showed that Daihuang Zhechong pill could increase the content of TNOs and reduce the content of iNOS in model rats. The experimental results of observing the effect of Daihuang Zhechong Pill on the ultrastructure of model rats showed that 
the intima of blood vessels in Daihuang Zhechong pill group was regular, the endothelium was relatively intact, some endothelial cells fell off, the continuity of blood vessel wall was good, and a few thrombosis were relatively unobstructed in the lumen. Under electron microscope, histology showed that in Daihuang Zhechong pill group, there were trace exudation and inflammatory cells under the middle membrane, or the middle layer was intact, the endothelium was continuous, there were few platelets aggregation in the lumen, and the cytoplasm of endothelial cells was full.

\subsection{Pharmacological research on prevention and treatment of atherosclerosis}

Lin Zhang et al. ${ }^{[3]}$ made a rat atherosclerosis model by high-fat diet combined with mechanical injury of abdominal aortic intima. After the experiment, the contents of NO and ET-1 in abdominal aorta of rats in each group were measured: Compared with the model group, the content of NO in Daihuang Zhechong pill group increased and the content of ET-1 in Daihuang Zhechong pill group decreased significantly. Previous studies have shown that: Endogenous NO can effectively combat atherosclerosis (AS), that is, endogenous NO is an internal anti AS molecule, and ET is a molecule with strong vasoconstriction. Therefore, the increase of NO content in rats will produce more anti AS molecules, which can significantly alleviate atherosclerosis. It is further confirmed that Daihuang Zhechong pill can effectively treat atherosclerotic diseases.

\section{Clinical application of Daihuang Zhechong pill}

\subsection{Treatment of hepatitis $B$ liver fibrosis}

Hongbin Chen ${ }^{[4]}$ selected entecavir combined with Daihuang Zhechong pill to diagnose and treat hepatitis B liver fibrosis. After treatment for a period of time, the degree of inflammatory activity and liver fibrosis in the two groups decreased to varying degrees, and the degree of liver fibrosis in the study group was significantly lower than that in the control group. It is proved that the combination of entecavir and Daihuang Zhechong pill is effective in the diagnosis and treatment of hepatitis B liver fibrosis. Hepatitis B liver fibrosis is the process from hepatitis B to cirrhosis. Therefore, clinically effective treatment of hepatitis B liver fibrosis can reasonably block the further development of the disease into cirrhosis, so as to fight hepatitis B virus, reduce inflammatory activities, improve microcirculation, and finally improve liver function.

\subsection{Treatment of chloasma}

Haiying Li et al. ${ }^{[5]}$ used Daihuang Zhechong pill to treat chloasma syndrome, which belongs to Qi stagnation and blood stasis type. After a period of time, observing the data of the two groups, we can draw a conclusion: The total effective rate of the treatment group was much higher than that of the control group. Traditional Chinese medicine believes that chloasma belongs to "blackish facial patch", "dusty complexion" and other diseases. Its pathogenesis involves liver, spleen and kidney. The total pathogenesis is blood deficiency and blood stasis. The effect of Daihuang Zhechong pill is to promote blood circulation and break blood stasis. Combined with the experimental results, it can be confirmed that Daihuang Zhechong pill has a good effect in the treatment of chloasma of Qi stagnation and blood stasis type.

\subsection{Treatment of chronic renal failure}

Bian Zhibin ${ }^{[6]}$ treated chronic renal failure with Daihuang Zhechong pill combined with western medicine. After treatment for a period of time, the levels of serum creatinine, blood urea nitrogen and blood uric acid in the two groups were significantly reduced, and the scores of low back pain and dark complexion in the two groups were significantly improved. Modern pharmacological studies have proved that hirudin in leeches can improve hemorheology, improve renal microcirculation, and reduce serum urea nitrogen and 
creatinine levels, so as to protect the kidney. Rhubarb aqueous extract can reduce the level of blood urea nitrogen and delay the grade of renal failure. Therefore, Daihuang Zhechong pill combined with western medicine can have a high clinical cure rate in the treatment of chronic renal failure.

\section{Funding}

Xianyang science and technology research plan project in 2018 (2018K02-84, study on the intervention mechanism of Daihuang Zhechong Pill on carotid atherosclerotic plaque)

\section{Disclosure statement}

The authors declare no conflict of interest.

\section{References}

[1] Liu XD, Zhao ZZ, Lu P, Xu XJ, 2018, Effect of Daihuang Zhechong Pill on BAMBI Expression in Rat Liver Tissue. Lishizhen Medicine and Materia Medica Research. 29(12): 2858-2861.

[2] Hui XY, Guo WG, Teng L, et al., 2018, Effects of Daihuang Zhezhuang Pill on TNOs, iNOS and Ultrastructure of Rat Deep Vein Thrombosis Model. Information on Traditional Chinese Medicine. 35(2): 47-50.

[3] Zhang L, Li DY, 2012, Randomized Controlled Study on the Effect of Daihuang Zhechong Pill on the Expression of NO and ET-1 in Atherosclerotic Rat Model. Information on Traditional Chinese Medicine. 4: 27-28.

[4] Chen HB, 2018, Clinical Effect of Entecavir Combined with Daihuang Zhechong Pill in the Treatment of Hepatitis B Liver Fibrosis. Clinical Research and Practice. 5: 133-134.

[5] Li HY, Dong H, Gao YZ, 2015, Clinical Observation of Daihuang Zhechong Pill in the Treatment of Chloasma of Qi Stagnation and Blood Stasis Type. Chinese and Foreign Medical Research. 13(18): 114-115.

[6] Bian ZB, 2018, Clinical Study of Daihuang Zhechong Pill Combined with Western Medicine in the Treatment of Chronic Renal Failure, China Foreign Medical Treatment. 13: 101-103. 